\title{
Determinación de la eficiencia productiva de cuatro abo- nos orgánicos y una fertilización química utilizando plantas de maíz (Zea mays) como indicadoras
}

\author{
Daniel Fernández Rivera \\ Estudiante de Agronomía, Universidad Estatal a Distancia; fernandez27agro@gmail.com \\ Tel: 8630- $9930 \cdot 2559-0469$
}

Recibido: 17 junio 2013

Aceptado: 05 julio 2013

\section{RESUMEN}

El objetivo de esta investigación fue evaluar la calidad y eficiencia nutricional de los abonos orgánicos: compost, lombricompost, bocashi, biofermento y una fertilización química en la variedad H5 de maíz. Se realizaron seis tratamientos (T), uno para cada abono orgánico, una fertilización química y un tratamiento testigo. Las variables analizadas fueron: altura de las plantas, diámetro de los tallos; el peso, largo y diámetro de las mazorcas. En la altura se presentaron diferencias estadísticamente significativas a un nivel de significancia del $0,05 \%$ y del $0,01 \%$ entre cada uno de los (T). En el caso de los diámetros no se presentaron diferencias estadísticamente significativas entre cada uno de los (T), a un nivel de significancia del $0,05 \%$ y $0,01 \%$. El mayor numero de mazorcas se alcanzo con el compost, con el biofermento se obtuvo el mayor peso y diámetro en las mazorcas.

Palabras claves: Maíz, bocashi, compost, lombricompost, biofermento, fertilizantes.

\begin{abstract}
The objective of this research was to evaluate the quality and nutritional efficiency of the organic fertilizers: compost, vermicompost, bokashi, bioferment and a chemical fertilization in the H5 corn variety. There were six treatments $(\mathrm{T})$, one for each organic fertilizer, a chemical fertilization and a control treatment. The analized variables were: plant height, stem diameter, weight, length and cob diameter. In plant height differences were statistically significant at a significance level of $0.05 \%$ and $0.01 \%$ between each treatment $(\mathrm{T})$. There were not statistically significant differences between each treatment $(\mathrm{T})$ in the diameters, at a significance level of $0.05 \%$ and $0.01 \%$. The greatest number of cobs was reached with the compost, the greatest weight and diameter of the cobs was obtained with the bioferment.
\end{abstract}

Key words: Corn, bokashi, compost, vermicompost, bioferment, fertilizers.

\section{Introducción}

La agricultura orgánica es un sistema de producción que trata de utilizar al máximo los recursos de la finca, dándole énfasis a la fertilidad del suelo, la actividad biológica y al mismo tiempo, a minimizar el uso de los recursos no renovables, no utilizar fertilizantes ni plaguicidas sintéticos para proteger el medio ambiente y la salud humana (Andersen, 2003).

El no utilizar fertilizantes químicos, implica la utilización de abonos orgánicos los cuales elaborados y utilizados de una manera adecuada, permiten producir cosechas satisfactorias. Los abonos orgánicos son enmiendas que se incorporan al suelo para mejorar sus propiedades físicas, químicas, biológicas y con ello su fertilidad (Leblanc et al, 2007).

En la sede regional del INA en Turrialba, se preparan cuatro tipos de abonos orgánicos: (compost, lombricompost, Bocashi, y biofermentos). Sin embargo, no existe suficiente información que permita constatar la eficiencia productiva de cada uno de estos abonos en un determinado cultivo. Entendiendo eficiencia productiva, como la capacidad de un abono para lograr un adecuado crecimiento de las plantas en sus distintas etapas fenológicas, es decir en las diferentes fases de crecimiento que atraviesa una planta hasta llegar a la cosecha.

Según Leblanc et al (2007), una forma práctica de avaluar la calidad y eficiencia de un abono orgánico es a través de ensayos de producción en campo, es decir evaluando a nivel de campo el crecimiento que presenta un determinado cultivo con la aplicación del abono orgánico. 
El maíz resulta ser un cultivo adecuado para utilizar como indicador, ya que absorbe una cantidad considerable de nutrientes, además de ser un cultivo muy adaptable a las condiciones agroclimáticas de la región y de sufrir menores daños por patógenos en comparación con otros cultivos.

El maíz es el cereal que más importancia ha tenido en varios sectores de la economía a escala mundial durante el siglo XX y en los inicios del XXI. En los países industrializados, el maíz se utiliza principalmente como forraje, materia prima para la producción de alimentos procesados y, recientemente, para la producción de etanol. Por el contrario, en algunos países de América Latina y, cada vez más en países africanos, un gran porcentaje del maíz que se produce o importa se destina al consumo humano (Serratos, 2009). En cuanto a la importancia del cultivo de maíz en nuestro país, Nevio Bonilla (2008) cita que este ha sido producido por pequeños productores $(85 \%)$ cuya área de siembra ha oscilado entre 1,50 y 3,00 hectáreas.

Por otro lado, el cultivo orgánico de maíz exige la utilización de abonos orgánicos, los cuales varían mucho en el contenido nutricional y características físicas. Considerando que el contenido de nutrientes varía para cada abono, es de esperar que si se utilizan diferentes tipos de abonos en el cultivo, los rendimientos podrían variar, ya que la cantidad de nutrientes aportada por cada abono es variable.

Matheus et al (2007) compararon tres abonos orgánicos (lombrimicompost, compost y gallinaza) y una fertilización química, en plantas de maíz sembradas en macetas. Los resultados indicaron que el mayor efecto del tratamiento con fertilización química fue en la fase inicial del ensayo, mientras que los tratamientos con productos orgánicos alcanzaron su mayor rendimiento a los 63 días, evidenciando su acción residual, lo cual es conveniente pues de este modo las plantas pueden aprovechar de mejor manera los nutrientes.

Martínez y Cantero (2002), compararon dos abonos orgánicos composteados (gallinaza y estiércol vacuno), con un fertilizante mineral (18-46-0) en el cultivo del maíz; Los resultados en cuanto a rendimiento, así como mayor tasa de retorno marginal, se obtuvieron con la aplicación de $2772.84 \mathrm{Kg} /$ ha de compost de gallinaza obteniéndose un rendimiento de $5848.86 \mathrm{~kg} / \mathrm{ha}$ y una tasa de retorno marginal de $662 \%$.

Relacionado con esto, en un ensayo elaborado por Marín y Báez (2010), se evaluó el efecto de una mezcla de abonos orgánicos en comparación con una fertilización sintética sobre el crecimiento y rendimiento del cultivo de maíz. Los resultados no indicaron diferencia significativa para las variables evaluadas decrecimiento y rendimiento; sin embargo, el manejo convencional presentó mayor rendimiento (de 20 kilogramos) con un valor ligeramente más alto de $4300 \mathrm{~kg}$ ha-1 contra $4280 \mathrm{~kg}$ ha-1del manejo orgánico.

\section{Materiales y métodos}

La investigación se realizó en el modulo agropecuario de la sede regional del Instituto Nacional de Aprendizaje (INA) en Turrialba, provincia Cartago, Costa Rica. La posición geográfica del cantón de Turrialba es la siguiente: O9 ${ }^{\circ} 47^{\prime} 14$ ' latitud norte y 830 34' 03' longitud oeste, a $600 \mathrm{msnm}$, con temperaturas promedio de 24 a $29^{\circ} \mathrm{C}$ y una precipitación promedio anual de $2600 \mathrm{~mm}^{3}$.

Se realizo un modelo de bloques completos al azar, con seis tratamientos y seis repeticiones. Para cada tratamiento se sembraron dos hileras de maíz, la dosis de los abonos se estimo con base en los resultados del análisis químico del suelo y de los abonos orgánicos, los tratamientos (T) fueron los siguientes:

T1= Testigo: No se aplicó ningún tipo de abono. T2= Bocashi, T3=Lombricompost, T4= Compost: Para cada uno de estos tratamientos se realizaron cinco aplicaciones de $100 \mathrm{~g}$ por planta fraccionadas de la siguiente manera: a la siembra, 15 días después de la siembra (dds), 30 dds, a los 45 dds y a los 60 dds.

T5= Biofermento: Este se aplico al $15 \%$ disuelto en agua, tanto a la raíz como al follaje, aplicando $500 \mathrm{ml}$ por planta a la siembra (únicamente al suelo), 15 dds, 30 dds, a los 45 dds y a los 60 dds (únicamente al suelo) . 
CUADRO A

Resultado del análisis químico de los abonos sólidos realizado en los laboratorios del INTA.

\begin{tabular}{lcccccccccccc}
\multicolumn{1}{c}{ Muestra } & $\mathrm{pH}$ & $\mathrm{Al}$ & $\mathrm{Ca}$ & $\mathrm{Mg}$ & $\mathrm{K}$ & $\mathrm{P}$ & $\mathrm{Zn}$ & $\mathrm{Mn}$ & $\mathrm{Cu}$ & $\mathrm{Fe}$ & $\mathrm{N}$ & $\mathrm{C}: \mathrm{N}$ \\
${ } }$ & 6,0 & 0,25 & 4,7 & 1,5 & 0,53 & 16 & 6,7 & 16 & 22 & 85 & 0,45 & 3,11 \\
Bocashi & 6,4 & 0,20 & 3,1 & 2,5 & 5,23 & 60 & 14,0 & 152 & 10 & 86 & 1,71 & 11,9 \\
Lombricompost & 6,4 & 0,20 & 6,7 & 5,2 & 6,83 & 159 & 18,9 & 11 & 6 & 87 & 1,62 & 23,4 \\
Compost & 6,5 & 0,20 & 6,2 & 4,5 & 6,02 & 94 & 4,3 & 25 & 7 & 26 & 1,54 & 13,0 \\
\hline
\end{tabular}

CUADRO B

Análisis químico del biofermento

\begin{tabular}{ccccccccc} 
& \multicolumn{4}{c}{ Porcentaje (\%) } & & \multicolumn{3}{c}{$\mathrm{mg} / \mathrm{kg}$} \\
$\mathbf{N}$ & $\mathbf{P}$ & $\mathbf{K}$ & $\mathbf{C a}$ & $\mathbf{M g}$ & $\mathbf{C u}$ & $\mathbf{Z n}$ & $\mathbf{M n}$ & $\mathbf{F e}$ \\
3,15 & 0,28 & 3,79 & 1,84 & 1,24 & 5 & 405 & 226 & 1056 \\
\hline
\end{tabular}

T6= Fertilización química: Se realizó en base a los resultados del análisis de suelo. Se aplicó $10 \mathrm{~g}$ por planta de la fórmula 10-30-10 a la siembra, $10 \mathrm{~g}$ por planta de la formula 12- 2412 a los 30 dds y $15 \mathrm{~g}$ de la fórmula 15-03-18 a los 60 dds.

El análisis químico del suelo y los abonos, se realizo en el laboratorio de suelos del Instituto Nacional de Transferencia de Tecnología Agropecuaria (INTA), En el cuadro "a" se indica el contenido nutricional del suelo y de los abonos compost, lombricompost y bocachi, en el cuadro "b" se indica el contenido de elementos del biofermento.

Para calcular la dosis requerida de los abonos, se realizaron conversiones para poder pasar el contenido de los nutrientes expresados en Cmol (+)/ (K, Ca y Mg) a porcentaje, los factores de conversión usados para cada uno de estos elementos, fueron los siguientes:
- $\quad \mathrm{K}: 0,0391$

- Ca: 0,020

- Mg:0,0121

Para los elementos expresados en mg/l, únicamente se dividió la cantidad entre 100 para pasar los valores a gramos. Para el caso del nitrógeno se considero el porcentaje de nitrógeno total $(\% \mathrm{~N})$ y la relación carbono nitrógeno $(\mathrm{C}: \mathrm{N})$.

Las cantidades de los elementos expresadas en gramos se compararon con la cantidad de nutrientes que extraen las plantas de maíz, las cuales se indican en el cuadro "c", considerando un rendimiento de 7,00 toneladas por hectárea (ton/ha).

Las variables analizadas fueron las siguientes: Altura de las plantas a los $15,30,45$ y 60 dds, diámetro del tallo a los 15, 30, 45 y 60 dds. Numero de mazorcas por tratamiento y numero de mazorcas por plantas en cada uno de los

CUADRO C

\section{Cantidad de elementos por las plantas de maíz en kilogramos por hectárea ( $\mathrm{Kg} / \mathrm{ha})$.}

\begin{tabular}{ccccccc}
$\begin{array}{c}\text { Rendimiento } \\
\text { Ton/ha }\end{array}$ & $\begin{array}{c}\mathrm{N} \\
\text { Total }\end{array}$ & $\begin{array}{c}\mathrm{P} \\
\mathrm{Kg} / \mathrm{ha}\end{array}$ & $\begin{array}{c}\mathrm{K} \\
\mathrm{Kg} / \mathrm{ha}\end{array}$ & $\begin{array}{c}\mathrm{Ca} \\
\mathrm{Kg} / \mathrm{ha}\end{array}$ & $\begin{array}{c}\mathrm{Mg} \\
\mathrm{Kg} / \mathrm{ha}\end{array}$ & $\begin{array}{c}\mathrm{S} \\
\mathrm{Kg} / \mathrm{ha}\end{array}$ \\
1,00 Ton & 40,00 & 9,00 & 33,00 & 8,00 & 5,00 & - \\
3,00 Ton & 72,00 & 16,00 & 45,00 & 4,00 & 4,00 & 5,00 \\
5,00 Ton & 170,00 & 30,00 & 60,00 & 23,00 & 25,00 & 20,00 \\
7,00 Ton & 200,00 & 34,00 & 130,00 & 31,00 & 24,00 & - \\
\hline
\end{tabular}

Fuente: (Bonilla, 2008). 
tratamientos. Peso de las mazorcas (olotes) al momento de la cosecha, diámetro y largo de las mazorcas al momento de la cosecha. Durante el análisis de cada una de las variables, se consideró una muestra de 10 unidades (plantas o mazorcas) para cada tratamiento.

Para cada una de las variables analizadas se calculo el valor promedio, y se realizo una comparación de los resultados de cada uno de los tratamientos. En el caso de la altura de las plantas y diámetro se verifico si existían diferencias estadísticamente significativas entre cada uno de los tratamientos aun nivel de significancia del $0,05 \%$ y del $0,01 \%$ utilizando una tabla de Duncan. La calidad de la cosecha, se analizo midiendo la altura, diámetro y peso de las mazorcas, se calcularon los valores promedios y se compararon entre cada uno de los tratamientos. Además, se analizaron las diferencias entre el número de mazorcas por tratamiento, y el número de mazorcas por planta para cada uno de los tratamientos, luego se compararon los resultados para determinar en cual tratamiento se cosecharon mas mazorcas y en cual se obtuvo un mayor número de mazorcas por planta.

\section{Resultados y discusión}

La altura de las plantas se empezó a medir a partir del día 15 (V5-V6) después de la siembra, las mediciones se realizaron desde la base del tallo hasta la punta de la última hoja formada, utilizando un flexómetro. En el cuadro d, se indica la altura promedio de las plantas a diferentes edades para cada uno de los tratamientos.

Tal como se observa en el cuadro d, los promedios en altura más bajos se obtuvieron en los (T) testigo y Bocashi. Siendo los promedios más altos los de los (T) los del biofermento y químico, sin embargo; a los 60 dds donde se realizo la última medición el compost presento la mayor altura promedio $(280,2 \mathrm{~cm})$. De los abonos orgánicos el biofermento fue el que presento mayor altura promedio hasta los 45 dds, al día 60 la altura fue menor que los (T) lombricompost, compost. De todos los tratamientos el químico fue el que presentó la mayor altura promedio en todas las edades de las plantas, hasta el día 45 ya que a partir del día 60 la altura fue similar a la presentada por los $(\mathrm{T})$ orgánicos.

La mayor altura hasta los 45 dds de las plantas fertilizadas con abono químico demuestra la

CUADRo D

Altura promedio de las plantas a diferentes edades para cada uno de los tratamientos

\begin{tabular}{ccccccc} 
Tiempoen(dds) & Testigo & Bocashi & $\begin{array}{c}\text { Lombri } \\
\text { Compost }\end{array}$ & Compost & Biofermento & Químico \\
15 & $12 \mathrm{~cm}$ & $13,4 \mathrm{~cm}$ & $15,89 \mathrm{~cm}$ & $15,7 \mathrm{~cm}$ & $19,15 \mathrm{~cm}$ & $23.5 \mathrm{~cm}$ \\
30 & $46,6 \mathrm{~cm}$ & $46,5 \mathrm{~cm}$ & $56,35 \mathrm{~cm}$ & $59,43 \mathrm{~cm}$ & $62,2 \mathrm{~cm}$ & $65,2 \mathrm{~cm}$ \\
45 & $109,8 \mathrm{~cm}$ & $111,55 \mathrm{~cm}$ & $129 \mathrm{~cm}$ & $144,5 \mathrm{~cm}$ & $158,3 \mathrm{~cm}$ & $160,4 \mathrm{~cm}$ \\
60 & $246,6 \mathrm{~cm}$ & $255,3 \mathrm{~cm}$ & $272.1 \mathrm{~cm}$ & $280,2 \mathrm{~cm}$ & $270,3 \mathrm{~cm}$ & $276,8 \mathrm{~cm}$ \\
\hline
\end{tabular}

dds: días después de siembra

CuAdRo E

Diámetro promedio de los tallos en diferentes días para cada uno de los tratamientos

\begin{tabular}{cccccccc} 
Edad en días & Testigo & Bocashi & lombricompost & compost & Biofermento & Químico \\
15 & $0,44 \mathrm{~cm}$ & $0,54 \mathrm{~cm}$ & $0,59 \mathrm{~cm}$ & $0,73 \mathrm{~cm}$ & $0,79 \mathrm{~cm}$ & $0,69 \mathrm{~cm}$ \\
30 & $1,66 \mathrm{~cm}$ & $1,70 \mathrm{~cm}$ & $2,07 \mathrm{~cm}$ & $2.33 \mathrm{~cm}$ & $2,32 \mathrm{~cm}$ & $2,21 \mathrm{~cm}$ \\
45 & $3,06 \mathrm{~cm}$ & $3,24 \mathrm{~cm}$ & $3,33 \mathrm{~cm}$ & $3,50 \mathrm{~cm}$ & $3,52 \mathrm{~cm}$ & $2,76 \mathrm{~cm}$ \\
60 & $3,16 \mathrm{~cm}$ & $3,13 \mathrm{~cm}$ & $3,19 \mathrm{~cm}$ & $3,38 \mathrm{~cm}$ & $3,33 \mathrm{~cm}$ & $2,91 \mathrm{~cm}$ \\
\hline
\end{tabular}


CUADRO F

Número de mazorcas para cada uno de los tratamientos según el número de plantas

\begin{tabular}{lcccccc}
\multicolumn{1}{c}{ Aspecto } & Testigo & Bocashi & Lombricompost & Compost & Biol & Químico \\
Número de plantas & 44 & 61 & 72 & 64 & 61 & 81 \\
Numero de mazorcas & 45 & 64 & 77 & 91 & 79 & 78 \\
Mazorcas por planta & 1,02 & 1,04 & 1,06 & 1,42 & 1,29 & 0,96 \\
\hline
\end{tabular}

rápida disponibilidad de nutrientes que este tipo de fertilizantes tienen, al contrario la poca diferencia presentada a los 60 dds revela la lenta disponibilidad de nutrientes que tienen los abonos orgánicos sólidos, lo cual trae muchas ventajas pues permite una disponibilidad de nutrientes durante periodos mas largos.

En el caso del biofermento al comparar las alturas promedio con los otros (T) orgánicos y con el (T) químico, hasta los 45 dds el biofermento fue superado solamente por el (T) químico, lo cual se debió probablemente a la rápida disponibilidad de nutrientes que tienen los biofermentos al ser aplicados de forma liquida.

Para realizar las mediciones del diámetro de los tallos se utilizó un vernier, estas se iniciaron a partir del día 15 y se hicieron en la base del tallo (en la superficie del suelo o/y en la parte superior de las raíces laterales). En el cuadro "e", se indican los diámetros promedios por planta a diferentes edades para cada uno de los tratamientos.

Según lo señalado en el cuadro "e", los promedios más altos se obtuvieron a los 30, 45 y 60 dds; sin embargo, a partir del día 60 los diámetros promedios disminuyeron. Hasta el día 60, el promedio más bajo fue para el tratamiento químico y el más alto para el compost.

Considerando los datos registrados hasta los 60 dds, es importante recordar que, en muchas especies de plantas, el tallo es además uno de los órganos de reserva de agua y foto asimilados especialmente con antelación a la etapa reproductiva (masbotanica.galeon.com), con base en esto, es posible que el diámetro de los tallos disminuyo a partir de los 45 dds, debido a que las sustancias de reserva (azucares almacenados) fueron utilizadas por las plantas al momento de la floración (R1), y es probable que por esta razón en los tratamientos orgánicos el diámetro de los tallos al día 60 disminuyó.

El Bocashi presentó los promedios más bajos en comparación con el resto de abonos orgánicos y el tratamiento químico. Sin embargo del día 30 al 45, las plantas fertilizadas con Bocashi presentaron el mayor incremento en 15 días el cual fue de $1,54 \mathrm{~cm}$, esto podría indicar que la disponibilidad de nutrientes se incrementó debido a la muerte microbiana.

El rendimiento en la producción considerando la cantidad de mazorcas por tratamiento, fue muy variable de un tratamiento a otro. En el cuadro "f", se indican la cantidad de mazorcas por tratamiento según el número de plantas.

La cantidad de mazorcas fue menor en el tratamiento testigo que para el resto de los tratamientos; sin embargo, el tratamiento químico presentó el menor número de mazorcas por planta, la mayor cantidad de mazorcas se logró en el tratamiento con biofermento y compost, en este último se alcanzó el mayor número de mazorcas por planta.

Por otro lado, el peso promedio de las mazorcas fue menor en el tratamiento testigo, que en el resto de los tratamientos, siendo los tratamientos con biofermento y compost los que alcanzaron

CuAdro G

\section{Pesos promedios de las mazorcas en cada uno de los tratamientos}

\begin{tabular}{lc}
$\quad$ Tratamiento & Peso promedio de las mazorcas \\
Testigo & $484,7 \mathrm{~g}$ \\
Bocashi & $516 \mathrm{~g}$ \\
Lombricompost & $574 \mathrm{~g}$ \\
Compost & $580,5 \mathrm{~g}$ \\
Biofermento & $590 \mathrm{~g}$ \\
Químico & $427 \mathrm{~g}$ \\
\hline
\end{tabular}


un mayor peso promedio de las mazorcas. En el cuadro "g", se indica el peso promedio de las mazorcas para cada uno de los tratamientos.

Las plantas fertilizadas con compost alcanzaron un peso promedio de las mazorcas aproximadamente de 95,8 g más que las del tratamiento testigo. Cuando se utilizó biofermento se obtuvo un peso promedio de $105,3 \mathrm{~g}$ mayor que en el tratamiento testigo, en el lombricompost el peso promedio de las mazorcas fue de 89,3 g mayor que el de las plantas donde no se utilizó ningún tipo de abono.

El tratamiento químico alcanzo el menor peso promedio de las mazorcas, el cual fue de $163 \mathrm{~g}$ menos que el tratamiento con biofermento. De los tratamientos orgánicos, el Bocashi fue el que presento un menor peso promedio de las mazorcas, el cual fue apenas de $31.3 \mathrm{~g}$ mayor que las del testigo y $74 \mathrm{~g}$ menor que el tratamiento con biofermento.

El largo de las mazorcas fue ligeramente menor en el tratamiento testigo, pero no se presentaron diferencias entre los tratamientos orgánicos. el tratamiento químico presentó un largo promedio de las mazorcas de $37,7 \mathrm{~cm}$, aproximadamente $5 \mathrm{~cm}$ menos que el presentado por los tratamientos orgánicos.

El diámetro de las mazorcas fue menor en el tratamiento testigo, siendo los tratamientos lombricompost, compost y biofermento, los que presentaron un mayor diámetro promedio de las mazorcas, los cuales fueron de 6,71; 6,74 y 6, 82 respectivamente. El mayor diámetro promedio de las mazorcas se alcanzó cuando se utilizó biofermento y el menor en el tratamiento testigo. La diferencia entre el tratamiento químico y el biofermento fue aproximadamente de $0,63 \mathrm{~cm}$.

\section{Conclusiones}

En la altura de las plantas de maíz si se presentaron diferencias estadísticamente significativas a un nivel de significancia del $0,05 \%$ y del $0,01 \%$ entre cada uno de los tratamientos. Las diferencias se debieron básicamente los (T) testigo y Bocashi, ya que, los (T) con compost y biofermento no presentan mayores diferencias con el (T) químico.

Para el diámetro de los tallos no se presentaron diferencias estadísticamente significativas entre cada uno de los (T), a un nivel de significancia del $0,05 \%$ y $0,01 \%$.

El (T) con compost presento el mayor numero de mazorcas por planta. El mayor peso promedio y diámetro promedio de las mazorcas se logro cuando se utilizo biofermento, el diámetro de las mazorcas fue igual en todos los (T) orgánicos y menor en los (T) testigo y químico.

\section{Recomendaciones}

La aplicación de biofermento al $15 \%$ se puede realizar en cualquier etapa fenológica del cultivo. Los abonos sólidos al tener una disponibilidad más lenta de los nutrientes, se recomienda aplicarlos desde la siembra hasta el inicio de la etapa de llenado de las mazorcas, pero fraccionando las aplicaciones.

Considerando un posible plan de fertilización orgánico para el cultivo del maíz en base a los resultados obtenidos, se recomienda; la aplicación de 100 g por planta de lombricompost , compost o bocashi a la siembra, $100 \mathrm{~g}$ por planta de compost a los 15 dds, una aplicación de biofermento diluido en agua al 15\% a los 15 dds, $100 \mathrm{~g}$ de compost o lombricompost por planta a los $30 \mathrm{dds}, 100 \mathrm{~g}$ de compost por planta a los 45 dds, una segunda aplicación de biofermento diluido en agua al 15\% a los $45 \mathrm{dds}$, y una tercera aplicación de biofermento diluido en agua al $15 \%$ a los 60 dds. En caso de utilizar Bocashi se recomienda usarlo durante los primeros $30 \mathrm{dds}$. 